\title{
Anisotropic flow in pp-collisions at the LHC
}

\author{
S.M. Troshin, N.E. Tyurin \\ Institute for High Energy Physics, NRC "Kurchatov Institute"
}

Protvino, 142281, Russian Federation

\begin{abstract}
We discuss collective effects in $p p$-collisions at the LHC energies and derive an upper bound for the anisotropic flow coefficients $v_{n}$. A possibility of its verification via comparison with the measurements of $v_{2}$ is considered. We use an assumption on the relation of the two-particle correlations with the rotation of the transient state of matter.
\end{abstract}




\section{Introduction}

It has appeared that the interaction of the protons exhibits similarity with interaction of nuclei, namely, the presence of certain collective effects was revealed in both cases. This point needs to be specified. The ridge-like structure has been observed in correlation function of the two secondary particles at RHIC in the peripheral collisions of nuclei (cf. for details [1] and references therein). It appeared that the two-particle correlations of the produced particles have a narrow distribution over $\Delta \phi$ (the relative azimuthal angle of the transverse momenta of the two particles) but wide distribution over $\Delta \eta$ (the pseudorapidity difference of the two detected particles). This phenomenon called a ridge effect is usually associated with collective properties of a medium produced under interaction of the nuclei.

The similar effect has also been revealed by the CMS Collaboration [2] in $p p-$ collisions at $\sqrt{s}=7 \mathrm{TeV}$ in the events with high multiplicities and it becomes evident that the form of correlations in pp-collisions resembles the form observed in AA-collisions. Later on, the ridge has been found in nuclear interactions at the LHC, in PbPb-collisions by ALICE, ATLAS and CMS [3, 4, 5]. We would like to emphasize here that inelastic peripheral collisions 1 are present in AA-collisions since the ions are extended objects. Inelastic peripheral collisions in $p p$ case are enhanced due to a reflective scattering mode [6] gradually turning on at the LHC. This enhancement is further amplified by the rescattering in $\mathrm{pPb}$-collisions where the ridge of a significantly higher magnitude that was observed at $\sqrt{s_{N N}}=5.02$ $\mathrm{TeV}$ [7].

Of course, one should expect a significant quantitative difference between $p p$ , $p A$ and $A A$-collisions and the signal of the ridge and other collective effects should be even much more conspicuous in $A A$-interactions due to a large size of both colliding objects.

The experimental results of RHIC and LHC on ridge in the two-particle correlation functions have demonstrated that the emergent hadronic matter is strongly correlated and reveals high degree of coherence. The similarities between the proton and nuclear collisions have been discussed for a long time ( $\mathrm{cf}$. e.g. [8], [9]).

It would be helpful to specify what the term of the reflective scattering mode means. In fact, the unitarity relation written in the impact parameter representation implies existence of the two scattering modes, which could be designated as the absorptive and the reflective ones. An attractive feature of the impact parameter representation is a diagonalization of the unitarity equation for the elastic

\footnotetext{
${ }^{1}$ It implies a presence of the nonvanishing orbital angular momentum in the initial collision events leading to particle production.
} 
scattering amplitude $f(s, b)$, i.e.

$$
\operatorname{Im} f(s, b)=|f(s, b)|^{2}+h_{\text {inel }}(s, b)
$$

at high energies with $\mathcal{O}(1 / s)$ precision [10], where $b$ is an impact parameter of the colliding hadrons. The term $|f(s, b)|^{2}$ is the elastic channel contribution, while the inelastic overlap function $h_{\text {inel }}(s, b)$ covers the contributions from the all intermediate inelastic channels. An elastic scattering $S$-matrix element is related to the amplitude $f(s, b)$ by the relation $S(s, b)=1+2 i f(s, b)$ and can be presented in the form

$$
S(s, b)=\kappa(s, b) \exp [2 i \delta(s, b)]
$$

with the two real functions $\kappa(s, b)$ and $\delta(s, b)$. The function $\kappa(0 \leq \kappa \leq 1)$ is a transmission factor, its value $\kappa=0$ corresponds to complete absorption. At high energies the real part of the scattering amplitude is small and can be neglected, allowing the substitution $f \rightarrow$ if .

The choice of elastic scattering mode, namely, absorptive or reflective one, is governed by the phase $\delta(s, b)$. The common assumption is that $S(s, b) \rightarrow 0$ at the fixed impact parameter $b$ and $s \rightarrow \infty$. It is called a black disk limit, and in this case the elastic scattering is completely absorptive. This implies the limitation $f(s, b) \leq 1 / 2$. There is another option: the function $S(s, b) \rightarrow-1$ at fixed $b$ and $s \rightarrow \infty$, i.e. $\kappa \rightarrow 1$ and $\delta=\pi / 2$. This limiting case is interpreted as a pure reflective scattering [6]. The principal point here is that $1 / 2<f(s, b) \leq 1$, as allowed by unitarity [11].

It is known that to probe experimentally the collective effects, one can use the anisotropic flows coefficients $v_{n}$ [12]. At the beginning we obtain an upper bound for the anisotropic flows which is based on the rational form of unitarization and discuss possible experimental measurements of the anisotropic flows in the second part of the note.

\section{Upper bound for the anisotropic flows in $p p$-collisions}

An appearance of the reflective scattering mode at the LHC energies is a key point for the derivation of the upper bounds for the anisotropic flows coefficients. The solution of the equation $S(s, b)=0$ separates the region where scattering is a pure absorptive one and the region where reflective scattering is present. It corresponds to the maximum value of $h_{\text {inel }}(s, b)=1 / 4$, and the derivative of $h_{\text {inel }}(s, b)$ has the form

$$
\frac{\partial h_{\text {inel }}(s, b)}{\partial b}=S(s, b) \frac{\partial f(s, b)}{\partial b}
$$

i.e. it equals to zero at $S(s, b)=0$. The sign of the derivative of the inelastic overlap function is opposite to the sign of $\partial f(s, b) / \partial b$ when $S(s, b)<0$. Thus, 
the central impact-parameter profile of the function $f(s, b)$ transforms into a peripheral one for the inelastic overlap function $h_{\text {inel }}(s, b)$. The same can be shown in a different way, namely, the function $h_{\text {inel }}(s, b)$ can be expressed as a product, i.e

$$
h_{\text {inel }}(s, b)=f(s, b)[1-f(s, b)] .
$$

If $f(s, b)>1 / 2$ at high energies and small impact parameters, then the function $h_{\text {inel }}(s, b)$ will have a maximum value of $1 / 4$ at non-zero impact parameter value. The inelastic overlap function in the reflective scattering mode $(S(s, b)<0)$ is affected by the self-damping of the inelastic channels contribution [13]. Thus, due to the reflective scattering the inelastic overlap function $h_{\text {inel }}(s, b)$,

$$
h_{\text {inel }}(s, b) \equiv \frac{1}{4 \pi} \frac{d \sigma_{\text {inel }}}{d b^{2}},
$$

has a peripheral dependence on the impact parameter with a peak at $b=r(s)$. Numerically, it happens in the region starting with energy $\sqrt{s} \simeq 2 \mathrm{TeV}$ [6].

A peripheral dependence of $h_{\text {inel }}(s, b)$ asymptotically leads to the approximate relation for any observable $A$, which describe a multiparticle production process. The relation (valid in the limit $s \rightarrow \infty$ ) has the form [14]:

$$
\left.A(s, \xi) \simeq A(s, b, \xi)\right|_{b=r(s)}
$$

since $A(s, \xi)$ ( $\xi$ is a variable or a set of variables), can be obtained from the corresponding impact-parameter dependent function $A(s, b, \xi)$ by integrating it with the weight function $d \sigma_{\text {inel }} / d b^{2}$. In Eq. (2) the function $r(s)$ is determined by the relation $S(s, b=r(s))=0$. Thus, at $b=r(s)$, by the definition of the function $r(s)$, the complete absorption of the initial elastic channel takes place.

Eq. (2) attributes the main role to the collision geometry and is applicable for the observables associated with the particle production processes at $s \rightarrow \infty$, where the reflective scattering being a dominating mode. The energy evolution from the central to the peripheral profile of the inelastic overlap function illustrates this point (Fig.1). In practice, Eq. (2) can be used with certain precaution for the mean multiplicity, average transverse momentum, anisotropic flows coefficients $v_{n}$ and multiplicity distribution $P_{n}(s)$ where the reflective scattering becomes noticeable, i.e. starting with the LHC energies. In general, the relative range of the variations of the impact parameter in the multiparticle production processes decreases with energy and the most typical inelastic event at very high energy is the event with a non-zero value of the impact parameter in the region in the vicinity of $b=r(s)$. The inelastic events at small and large impact parameter values are strongly suppressed at very high energies. The energies, where suppression of the inelastic production in the head-on collisions is strong, lie beyond the LHC energy range. 

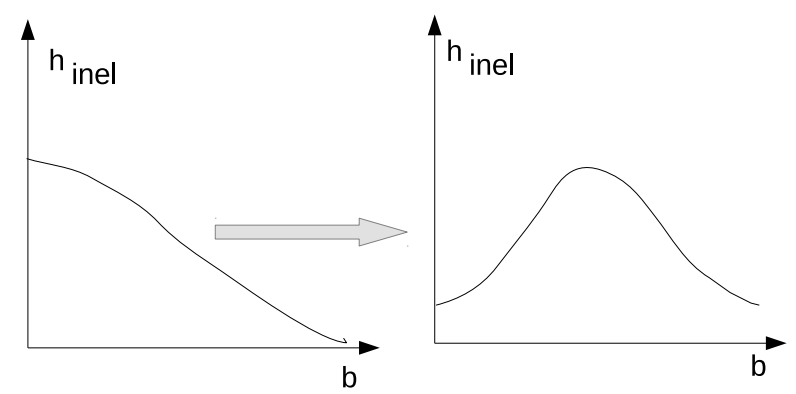

Figure 1: Evolution of the inelastic overlap function from a central to a peripheral profile with increasing center-of-mass energy.

After these preliminaries, we consider a bound for the anisotropic flows. There are several experimental probes of collective dynamics. A most widely discussed ones are the anisotropic flows [12] are determined by the equation

$$
v_{n}\left(p_{T}\right) \equiv\langle\cos (n \phi)\rangle_{p_{T}}
$$

which is the n-th Fourier moment of the azimuthal angle distribution of the particles with a fixed value of $p_{T}$. The angle $\phi$ is the azimuthal angle of the detected particle transverse momentum with respect to the reaction plane, i.e. the plane spanned by the collision axis $z$ and the impact parameter vector $\mathbf{b}$. Averaging is taken over large number of the inelastic events. By definition, the absolute value of coefficients $v_{n}\left(p_{T}\right)$ cannot exceed unity. It is a trivial upper bound. We will show in what follows that this bound can be reduced by factor of 4 due to account of unitarity for the inclusive cross-section.

The inclusive cross-section for unpolarised particles being integrated over impact parameter $\mathbf{b}$, does not depend on the azimuthal angle of the detected particle transverse momentum. It can be written with account for $s$-channel unitarity in the following form

$$
\frac{d \sigma}{d \xi}=8 \pi \int_{0}^{\infty} b d b \frac{I(s, b, \xi)}{[1+U(s, b)]^{2}} .
$$

Eq. (4) has been obtained in the $U$-matrix approach to unitarity when the elastic scattering $S$-matrix element is written in the rational form in the impact parameter representation:

$$
S(s, b)=\frac{1-U(s, b)}{1+U(s, b)},
$$

where $U(s, b)$ is the generalised reaction matrix element, corresponding to the elastic $2 \rightarrow 2$ scattering. It is considered to be an input dynamical quantity similar to the respective eikonal function. Since we consider the case of the pure 
imaginary scattering amplitude, the function $U$ should be pure imaginary too, i.e. the replacement $U \rightarrow i U$ has to be performed. The function $I(s, b, \xi)$ is an analog of the function $U(s, b)$ for reactions with particle production (cf. [15] and references therein) and $\xi$ is the set of kinematical variables ascribing the final detected particle.

The absolute value and direction of the vector $\mathbf{b}$ is the main issue under the determination of the anisotropic flows in $p p$-collisions. This vector can be controlled experimentally in the heavy ion collisions, but the situation, in general, is not explicitly clear in proton collisions. However, when we are approaching the high energy limit the magnitude $b=|\mathbf{b}|$ tends to be fixed, cf. Eq. (2), at $b=r(s)$. This fact and unitarization allows one to reduce upper bound for $v_{n}$ by the factor of 4 . It should be noted that the impact parameter $\mathbf{b}$ is the variable conjugated to the transferred momentum $\mathbf{q} \equiv \mathbf{p}_{a}^{\prime}-\mathbf{p}_{a}$ between two incident channels which describes production processes of the same final multiparticle state.

In the case when the impact parameter vector $\mathbf{b}$ and transverse momentum $\mathbf{p}_{T}$ of the detected particle are known the function $I$ in Eq. (4) does depend on the azimuthal angle $\phi$ between two vectors $\mathbf{b}$ and $\mathbf{p}_{T}$. The dependence on the azimuthal angle $\phi$ can be written in explicit form through the Fourier series expansion

$$
I\left(s, \mathbf{b}, y, \mathbf{p}_{T}\right)=\frac{1}{2 \pi} I_{0}\left(s, b, y, p_{T}\right)\left[1+\sum_{n=1}^{\infty} 2 \bar{v}_{n}\left(s, b, y, p_{T}\right) \cos n \phi\right] .
$$

The function $I_{0}(s, b, \xi)$ satisfies to the following sum rule

$$
\int I_{0}\left(s, b, y, p_{T}\right) p_{T} d p_{T} d y=\langle n\rangle(s, b) U(s, b),
$$

where $\langle n\rangle(s, b)$ is the mean multiplicity produced in collision with the impact parameter $b$. Therefore, the bare anisotropic flow coefficients $\bar{v}_{n}\left(s, b, y, p_{T}\right)$ are related to the measured those $v_{n}$ as follows

$$
v_{n}\left(s, b, y, p_{T}\right)=w(s, b) \bar{v}_{n}\left(s, b, y, p_{T}\right) .
$$

where the function $w(s, b)$ is

$$
w(s, b) \equiv[1+U(s, b)]^{-2}=[1+S(s, b)]^{2} / 4 .
$$

The variable $y$ denotes rapidity, i.e. $y=\sinh ^{-1}(p / m)$, where $p$ is a longitudinal momentum of the particle.

According to Eq. (2), we should fix the impact parameter value at $b=r(s)$ in the high energy limit. Since $\left.2 w(s, b)\right|_{b=r(s)}=1 / 4$ and

$$
\bar{v}_{n}\left(s, b, y, p_{T}\right) \leq 1
$$

\footnotetext{
${ }^{2}$ Note that $\left.S(s, b)\right|_{b=r(s)}=0$.
} 
we obtain

$$
v_{n}\left(p_{T}\right) \leq 1 / 4
$$

valid in the high energy limit. This relation provides information on the possible magnitude of the anisotropic flow coefficients in proton collisions. In the case of $p p$-scattering the respective experimental data are not yet available, but in the nuclear collisions the values of $v_{2}$, for example, at various energies are in the region $0.1-0.2$ at $p_{T} \sim 1.5 \mathrm{GeV} / \mathrm{c}$, i.e. not far below than 0.25 . The size of $v_{n}$ is evidently an energy-dependent in the case of $p p$-collisions in the approach and one could expect its increase with energy since orbital angular momentum increases with energy too. Therefore, the bound restricting this increase of $v_{n}$ can be rather useful.

The natural question is on the possibility to check the above bound experimentally, i.e. how to measure $v_{n}$ in the experiments with high energy proton beams. We will address this question considering for an example the case of the elliptic flow coefficient $v_{2}$. It is closely related to the reaction plane fixing in the particular inelastic event and will be discussed in the next section.

\section{The ridge and possibility to measure the anisotropic flow coefficients at the LHC with protons}

Elliptic flow coefficient $v_{2}$ can be expressed in a covariant form in terms of the impact parameter and transverse momentum vectors as follows

$$
v_{2}\left(p_{T}\right)=\left\langle\frac{\left(\hat{\mathbf{b}} \cdot \mathbf{p}_{T}\right)^{2}}{p_{T}^{2}}\right\rangle-\left\langle\frac{\left(\hat{\mathbf{b}} \times \mathbf{p}_{T}\right)^{2}}{p_{T}^{2}}\right\rangle,
$$

where $\hat{\mathbf{b}} \equiv \mathbf{b} / b$.

It is useful to recall what is known about this observable from results of nuclear collision experiments. The differential elliptic flow coefficient $v_{2}\left(p_{T}\right)$ increases with $p_{T}$ at small values of transverse momenta, then it becomes flatter in the region of the intermediate transverse momenta and decreases at large $p_{T}$. The integrated elliptic flow coefficient $v_{2}$ at high energies is positive and increases with $\sqrt{s_{N N}}$.

The particular production mechanism leading to appearance of the ridge [16] will be used in what follows. This mechanism is based on the geometry of the overlap region in proton collisions and dynamical properties of the produced transient state 3 . It assumes deconfinement at the initial stage of interaction. The geometrical picture of hadron collision at non-zero impact parameters implies [15] that the generated massive virtual quarks in the overlap region could obtain a large

\footnotetext{
${ }^{3}$ Of course, this is not a unique mechanism leading to the appearance of the ridge, cf. [17]
} 
initial orbital angular momentum at high energies. Due to the presence of the strong interaction between the quarks this orbital angular momentum would lead to a coherent rotation of the quark system located in the overlap region. The model estimates of the magnitude of this rotation are based on the following assumptions [16]. The rotation plane coincides with the reaction plane spanned over vectors $b$ and the initial particle momentum. It is similar to the rotation of the liquid where strong correlations between particles momenta exist and make their momenta to be coplanar. Therefore, the non-zero orbital angular momentum would be realised as a coherent rotation of the transient state as a whole. This state is supposed to be a quark-pion liquid. The arguments in favour of this claim can be found in [16]. Finally, the hadronization dynamics forms a colorless multiparticle final state.

The essential point needed for the existence of the matter rotation is a nonzero impact parameter in the collision. Of course, this rotation as well as the ridge effect coexist with other particle production mechanisms and rotation contributes to the $x$-component 4 of the transverse momentum, i.e. $p_{x}=p_{0}+\Delta p_{x}$ and does not contribute to the $y$-component, $p_{y}=p_{0}$.

It can also be supposed that the constituent quark number scaling [18] observed in heavy-ion collisions at intermediate values of $p_{T}$ and reflecting quark coalescence under the hadronization is to be valid in the proton collisions too, i.e. the following relation takes place

$$
v_{2}\left(p_{T}\right) \simeq n_{Q} v_{2}^{Q}\left(p_{T} / n_{Q}\right),
$$

$n_{Q}$ stands for the number of valence quarks in the final hadron and $v_{2}^{Q}$ is the elliptic flow coefficient of the quark $\mathrm{Q}$. The above relation can provide indication on the possible values of the constituent quark elliptic flow in the region of the intermediate $p_{T}$ values. Assuming validity of Eq. (10), the upper bound 1/12 can be obtained for $v_{2}^{Q}$ from the upper bound for $v_{2}$. The scaling given by the Eq. (10) can be extended into the region of small values of $p_{T}$ if $v_{2}$ is plotted versus transverse kinetic energy $K E_{T}=m_{T}-m$ (cf. [19]) and is closely related to the mechanism of hadron formation in the final state.

As it was noted earlier, the peripheral nature of the inelastic $p p$-interactions is controlled dynamically by the reflective scattering mode. This mode gradually turns on at the LHC energies [6, 20, 21]. Its appearance depends on the collision energy. But, the form of $h_{\text {inel }}(s, b)$ is only slightly different from a central one at the center-of-mass energy $\sqrt{s}=7 \mathrm{TeV}$. The value of $r(s)$ is about $0.2 \mathrm{fm}$ at this energy. Thus, since the highest number of particles are produced at the small impact parameters 5 , the ridge is observed in the events which have high multiplicity at $\sqrt{s}=7 \mathrm{TeV}$. With increase of the center-of-mass energy, the maximum of

\footnotetext{
${ }^{4}$ This component is directed along the vector $\mathbf{b}$.

${ }^{5}$ This is a standard assumption of the geometrical approach.
} 
$h_{\text {inel }}(s, b)$ will be shifted to the higher values of $b$ and form of $h_{\text {inel }}(s, b)$ would become more peripheral. This would lead to transition of the ridge to the events with average multiplicities. This prediction can be tested at the LHC energy $\sqrt{s}=13$ $\mathrm{TeV}$.

It should also be noted, that ridge effect can not be observed at the energies $\sqrt{s} \leq 2$ since there is no reflective scattering in the $p p$-collisions at such energies. The same reason leads to the vanishing coefficients of the anisotropic flow $v_{n} \simeq 0$ in the the $p p$ - (and $p A$-)collisions at those energies. This is a distinctive feature of this mechanism. It can be tested at RHIC.

The idea of rotating transient state can serve as a possible qualitative interpretation of the ridge and double-ridge structure observed by CMS, ALICE and ATLAS. The narrowness of the two-particle correlation distribution over the azimuthal angle is an important feature of the mechanism. However, it does not mean that the other options are not able to reproduce this dependence. It would be useful to have a quantitative method to analyze the magnitude of the rotation of the system.

It was said that the rotation plane coincides with the reaction plane. Thus, one can determine the normal to this plane being the unit vector

$$
\hat{\mathbf{n}}=\frac{\mathbf{p}_{1} \times \mathbf{p}_{2}}{\left|\mathbf{p}_{1} \times \mathbf{p}_{2}\right|}
$$

Since vectors $\hat{\mathbf{n}}$ and $\hat{\mathbf{b}}$ are orthogonal, one can rewrite the elliptic flow in the form

$$
v_{2}\left(p_{T}\right)=-\left\langle\frac{\left(\hat{\mathbf{n}} \cdot \mathbf{p}_{T}\right)^{2}}{p_{T}^{2}}\right\rangle+\left\langle\frac{\left(\hat{\mathbf{n}} \times \mathbf{p}_{T}\right)^{2}}{p_{T}^{2}}\right\rangle,
$$

The only experimentally measurable quantities enter Eq. (12). The vectors $\mathbf{p}_{1}$ and $\mathbf{p}_{2}$ are the momenta of particles whose distribution leads to the ridge effect. Therefore, tagging the events corresponding to the ridge effect should be performed during the analysis. Thus, the three-particles' correlations obtained during second stage of the analysis could be sensitive to and provide an information on the elliptic flow coefficient $v_{2}$. The above consideration has a shortcoming since it is based on the particular model explanation of the ridge effect in $p p$-collisions. However, it allows to estimate roughly the values of $v_{2}\left(p_{T}\right)$, i.e. together with the upper bound for the flow coefficients one can get hints on the magnitude of the collective effects at the LHC. The above bound is the main issue discussed in this note.

It should be noted that since the rotating matter involves electrically charged constituent quarks, one can expect that direct photons are to be produced by the relativistic synchrotron radiation mechanism and will have similar values of the anisotropic flows coefficients as the respective ones of the secondary hadrons. The 
rotation of the charged quasiparticles could give a straightforward way to the large elliptic flow of the direct photons at the LHC energies.

The use of the relativistic synchrotron radiation to explain direct photon elliptic flow at RHIC was suggested in [22]. As a dynamical reason for such radiation it was proposed to consider confinement phenomena.

\section{Conclusion}

The main point we would like to stress here is that the obtained upper bound for the anisotropic flows can serve as an indicator of the possible magnitude of these observables in $p p$-collisions. We would also like to note that the ridge effect is expected to become more conspicuous. This is due to the expected growing peripherality of the inelastic collisions with the center-of-mass energy. According to the above consideration, this effect should be observed in the events with multiplicities which are more close the the average values as the center-of-mass energy increases. One could expect that this transition to lower multiplicities can be exhibited already at $\sqrt{s}=13 \mathrm{TeV}$ where higher luminosities would be reached in $p p$-collisions. This is a distinctive feature of the ridge effect explanation. The upgrade of the LHC would provide additional possibilities for studying the collective effects in $p p$-collisions and the proposed mechanism.

\section{Acknowledgement}

We are grateful to A.M. Snigirev for bringing to our attention the phenomena of the large direct photon elliptic flow.

\section{References}

[1] STAR Collaboration, B. I. Abelev et al., Phys. Rev. Lett. 105, 022301 (2010).

[2] CMS Collaboration, V. Khachatryan et al., JHEP 1009, 091 (2010).

[3] ALICE Collaboration, K. Aamodt et al., Phys. Lett. B708, 249 (2012).

[4] ATLAS Collaboration, G. Aad et al., Phys. Rev. C86, 014907 (2012).

[5] CMS Collaboration, S. Chatrchyan et al., Eur. Phys. J. C72, 2012 (2012).

[6] S.M. Troshin, N.E. Tyurin, Int. J. Mod. Phys. A 22, 4437 (2007).

[7] CMS Collaboration, S. Chatrchyan et al., Phys. Lett. B718, 795 (2013). 
[8] R.M. Weiner, Int. J. Mod. Phys. E 15, 37 (2006).

[9] D. d'Enterria et al. Eur. Phys. J. C 66, 173 (2010).

[10] M.L. Goldberger and K.M. Watson, Collision Theory John Wiley and Sons, Inc., New-York—London—Sydney 1964.

[11] S.M. Troshin, N.E. Tyurin, Phys. Lett. B 316, 175 (1993).

[12] J.-Y. Ollitrault, Phys. Rev. D46, 229 (1992).

[13] M. Baker, R. Blankenbecler, Phys. Rev. 128, 415 (1962).

[14] S.M. Troshin, N.E. Tyurin, Int. J. Mod. Phys. A 29, 1450151 (2014).

[15] S.M. Troshin, N.E. Tyurin, Int. J. Mod. Phys. A 26, 4703 (2011).

[16] S.M. Troshin, N.E. Tyurin, Int. J. Mod. Phys. E 17, 1619 (2008).

[17] Wei Li, Mod. Phys. Lett. A 27, 1230018 (2012).

[18] S.A.Voloshin, Nucl. Phys. A 715, 379c (2003).

[19] M. He, R.J. Fries, R. Rapp, Phys. Rev. C 82, 034907 (2010).

[20] P. Desgrolard, L.L. Jenkovszky, B.V. Struminsky, Phys. Atom. Nucl. 63 (2000) 891.

[21] A. Alkin et al. Phys.Rev. D 89 091501(R) (2014).

[22] V.V. Goloviznin, A.M. Snigirev, G.M. Zinovjev, JETP Lett. 98, 61 (2013). 